\title{
Improving acute eye consultations in general practice: a practical approach
}

\author{
Michelle Ai Ling Teo
}

Lincolnshire NHS Trust, England

\begin{abstract}
There is significant evidence that patients with acute eye symptoms are poorly assessed in primary care. There is a tendency to diagnose viral or bacterial conjunctivitis in any acutely red eye. This has led to delays in treatment and in some cases, permanent loss of sight.
\end{abstract}

The aim of this project was to improve acute eye consultations within the Birchwood Medical Practice. The project focused on the "red flag" findings that would identify patients who require referral for same-day ophthalmology assessment.

A retrospective baseline audit was carried out on all cases read-coded "conjunctivitis" over the period of one year. Initially, only $2.8 \%$ of consultations had documented all four findings.

By considering the main factors that lead to poor eye assessments, two main areas for improvement were identified. These were education (reinforced with memory aids) and improving the availability of eye examination equipment within each consultation room. An "eye examination kit" was developed with the needs of the general practitioner in mind.

The practice was re-audited six weeks following the intervention. Consultations where all four red flag findings were documented rose from $2.8 \%$ to $50 \%$. This was found to be a statistically significant difference $(p<0.01)$. Pain was checked $63 \%$ of the time, compared to $26 \%$ prior to intervention. Visual acuity screening had increased to from $35 \%$ to $69 \%$. Photophobia was the most significantly increased metric, from being documented only $6 \%$ of the time to now $63 \%$ of the time. Documentation of whether the symptoms were unilateral or bilateral had also increased from $88 \%$ to $94 \%$ of consultations.

The initial audit indicated that general practitioners often diagnosed conjunctivitis without screening for symptoms of sight-threatening disease. However, it was clear from the results that the doctors had made a significant change to their approach to acute eye consultations. This shows that doctors are willing to make changes to their behaviour when given the right tools and information. It was felt that a first-hand understanding of the problem and an open discussion regarding the changes required was key to the success of this project.

This project has shown that significant improvements can be achieved with practical and inexpensive interventions. Therefore, general practices throughout the UK are encouraged to adopt similar strategies to improve the identification of patients needing same-day Ophthalmology assessment.

\section{Problem}

Patients with acute ophthalmic symptoms often present first to their general practitioner (GP). Multiple studies have shown that misdiagnosis of acute eye disease by GPs were not uncommon.[1,2] A survey of over 8000 UK GPs found that $68 \%$ affirmed the statement that they had "some uncertainties about eyes", while $10 \%$ admitted to being "scared stiff of eyes".[3]

Despite evidence that there is substandard management of acute eye cases in primary care, little is known about how GPs investigate and diagnose cases of an acutely red eye. This project aims to improve the quality of acute eye consultations with simple yet highyield interventions, such that they would be applicable at any NHS general practice.

\section{Background}

The Birchwood Medical Practice is a large general practice serving the area of Birchwood, Lincoln. It has a practice population of 9640 patients. Although the practice routinely carries out clinical audits, there has never been an ophthalmology-focused review.

Eye consultations make up one in 50 GP consultations.[4] This translates to roughly four cases seen a week per GP, with some GPs reporting up to 10 or more a week.[3] This means that eye consultations make up a significant proportion of all GP consultations.

A large study of 1062 acute eye cases showed that viral conjunctivitis was the most common misdiagnosis made by GPs [1]. In the same study, 11 patients were identified as having a "preventable severe adverse outcome" which included permanent visual loss. Ten out of these 11 cases were initially misdiagnosed as conjunctivitis.

Conjunctivitis has also been found in several studies to be the most 
common GP diagnosis in acute eye consultations.[2,5] As a result, it was determined that conjunctivitis cases would be an ideal sample to investigate the quality of eye consultations.

\section{Baseline measurement}

All cases read-coded "Conjunctivitis" over the period of one year (22nd August 2013 to 22nd August 2014) were audited. Baseline data were collected retrospectively and extracted using SystmOne Clinical Reporting Software. Only cases that were seen by doctors at Birchwood Medical Practice were included. This excluded cases seen at the walk-in out-of-hours GP, and patients whose records were transferred from another practice after moving into the area. After exclusion, 144 cases were included in this study.

Each consultation was audited against the NICE CKS guidelines for red eye [6] and conjunctivitis [7].The guidelines state that the history and findings (including negative findings) should always be documented. The important findings identified by NICE were:
1. Pain
2. Photophobia
3. Visual acuity
4. Whether the condition is unilateral or bilateral.

These symptoms almost always indicate a more serious eye disease rather than uncomplicated conjunctivitis.[1] If any of these red flag symptoms are present, the GP should consider referral of the patient for same-day assessment by ophthalmology.[6] Therefore the standard set for this audit was that $100 \%$ of consultations should have documented that the above symptoms were checked. An equipment audit was also carried out by distributing a questionnaire to assess the adequacy of eye equipment in each consultation room.

Only four (2.8\%) of the 144 consultations had all four symptoms checked. Whether or not the patient was experiencing pain or "soreness" in the eye was only recorded $26 \%$ of the time. Visual acuity or mention of "vision" was only recorded $35 \%$ of the time. Use of a Snellen chart to assess visual acuity was rarely recorded.

Photophobia was only screened for $6 \%$ of the time, indicating that this symptom may be missed. When discussed at the practice meeting, one of the general practitioners stated that she would write 'PEARLA' to indicate that there was no photophobia, as this clinical examination would involve shining light into the patient's eyes. This led to a discussion regarding the need to document symptoms in a way that can be understood by other practitioners that was consistent for medical, legal, and audit purposes.

Some indication of whether the symptoms were unilateral or bilateral was recorded in $88 \%$ of the consultations. This included consultations that mentioned if the symptoms were in the left or right eye, or in both eyes. Only a small proportion of the consultations actually stated the words "unilateral or bilateral." This means that in $12 \%$ of the consultations there was no indication whether the word "eye" meant the left or right eye, or both eyes. See figure 1 and 2 for a visual representation of these results.
Among the cases audited, one patient suffered an adverse event (prolonged pain and delayed treatment) due to being initially misdiagnosed as conjunctivitis. The patient presented with symptoms of a unilateral painful red eye. The attending doctor, a Foundation Year 2 trainee, came to a diagnosis of conjunctivitis and prescribed chloramphenicol eye drops. Three days later, the patient re-presented to another doctor who screened for pain, reduced visual acuity and photophobia. It was noted also that the symptoms were unilateral. It was also found that the patient was in fact, sensitive to light. The doctor carried out fluorescein staining and found a corneal abrasion. This patient was then referred to ophthalmology and started on ofloxacin eye drops. This missed case of a potentially sight-threatening condition highlights the importance of screening for the red flag symptoms and excluding possible differentials before coming to a diagnosis.

All the general practitioners admitted to infrequent use of the Snellen chart, with $40 \%$ of the GPs stating that this was simply because there was no Snellen chart in their consultation room. Of the GPs that did have a Snellen chart, $80 \%$ did not have the correct distance marked out. The survey of ophthalmoscopes indicated that all GPs had a working ophthalmoscope, which was reassuring.

See supplementary file: ds4273.docx - "Cycle 1 - bar charts and tables"

\section{Design}

In designing the interventions for this project, the factors which lead to poor acute eye consultations were first considered. Two main issues were identified. Firstly, there is significant anecdotal evidence among doctors, and also published studies [8] that have implied that undergraduate ophthalmology education in the UK is inadequate. This has left many non-ophthalmologists with a poor understanding and confidence in assessing acute eye problems.

Secondly, it was obvious that there was inconsistent access to eye examination equipment at the practice, so two main areas were identified for improvement. These were education (reinforced with memory aids) and improving the availability of eye examination equipment within each consultation room.

\section{Strategy}

PDSA cycle 1: The concept of this project was discussed with the GP partners at the practice. It was identified that the practice needs to understand the areas for improvement in their acute eye consultations so they are able to target changes. Therefore a baseline audit was planned, and it was agreed that a project looking into acute eye consultations would be useful. A search for all patients presenting with a "red-eye" was done on the SystmOne Clinical Reporting software. This search found only six cases coded with "red-eye" over the span of one year.

This was discussed with the practice administrator. It was suggested that an audit focusing on one particular diagnosis would be yield a larger sample. It was also then decided that a project to 
improve the eye equipment at the practice would also be useful.

PDSA cycle 2: Research into several studies of acute eye consultations in general practice highlighted that conjunctivitis was the most common ophthalmic misdiagnosis made by GPs. This focused the audit to cases given the diagnosis of "conjunctivitis" at the practice. A retrospective audit was therefore undertaken and the baseline results were presented at a practice meeting.

All full-time doctors (including GP consultants, registrars, and foundation trainees) and two senior nurse practitioners attended the meeting. The presentation began first with a short teaching session explaining the reasoning behind the recommendations by NICE.

An interactive approach was taken during the teaching. The attendees were presented with images of acutely red eyes and asked to describe the abnormalities found. In most cases it was difficult to come to a diagnosis without further information. However, each of the sight-threatening cases always presented with one or more of the red flag symptoms. This made the point that it is difficult to assess an acutely red eye based on examination alone, especially without the use of specialised equipment. It was therefore agreed that close attention should be paid to the symptoms of an acutely red eye.

The NICE guidelines for the acutely red eye were highlighted, which indicate the need to consider the six serious sight-threatening conditions. This included:
1. Keratitis
2. Iritis
3. Acute glaucoma
4. Scleritis
5. Penetrating injury or foreign body
6. Acid/alkali burns.

It was highlighted that benign conditions should not present with symptoms of pain, photophobia, or reduced visual acuity. It was stressed that if any of these symptoms were present, the above conditions should be considered. If appropriate, the patient should be referred for same day ophthalmology assessment.

This interactive approach allowed attendees to come to their own conclusions regarding the importance of these symptoms, instead of simply being told that this was a NICE guidelines requirement. This understanding was deemed important for success, as one of the main challenges of quality improvement projects is changing a person's behaviour.

PDSA cycle 3: A group discussion was carried out, which highlighted the pitfalls of acute eye assessments. One issue that came up was that no one was sure where the eye equipment would be. Some of the equipment was kept in the nurse's treatment room (e.g. saline drops and fluorescein strips), and some of the equipment was kept in the main storeroom. It was put forward that one would more likely perform fluorescein staining if they had easy access to the appropriate equipment in their consultation room.
Therefore, an eye examination kit was developed and made (figure 5) based on suggestions from the doctors at the practice. Its purpose was to encourage a more thorough eye examination, simply because the equipment required was readily available to them. Each eye kit included:
1. Near vision acuity chart
2. Pinhole
3. Pen torch with blue filter
4. Saline eye drops
5. Fluorescien strips
6. A red object (Neurotip)
7. Red flag symptoms card (described below).

The eye kits were trialed initially by the author for one week. Several issues were found early by using the eye kit in actual clinics. One issue was that there was little room for storage in the consultation rooms. Therefore a slimmer container was selected to store the relevant equipment. It was then difficult to lift the near vision acuity chart from the bottom of the smaller container, so a corner was fashioned to allow easy removal of the chart.

The eye kits were then distributed among all the 10 consultation rooms. Initial feedback from the doctors showed that they feel the eye kits were a useful addition to their consultation room equipment, as it kept everything needed in one place. It was also discussed with the practice manager that more Snellen charts needed to be ordered. The existing Snellen charts were marked out at the correct distance to allow for accurate visual acuity testing.

PDSA cycle 4: Following the practice meeting, it was clear that there was awareness that several red-flag symptoms should be checked in acute eye consultations. However, in further discussions, colleagues at the practice found it difficult to remember all four symptoms without being reminded. Feedback showed that the practice doctors particularly appreciated an acronym that would help them remember the four 'red-flag symptoms'. The acronym "P.A.L.S" was introduced, which stood for:

Pain

Acuity

Light (photophobia)

Side (unilateral or bilateral).

A printed card of the above acronym was placed on top of each eye kit to allow quick reference (figure $5 \mathrm{~b}$ ). It was felt that the placement of a physical card within each consultation room would act as a long term reminder for the doctors.

PDSA cycle 5: The positive feedback in support of memory aids encouraged the creation of an electronic template. The Birchwood Medical Practice has created several of their own templates which are particularly useful in guiding new trainees working at the practice. 
Several drafts of a red-eye template were created but were rejected as they were not compatible with the practice's computer system. Although the system does allow some flexibility, every symptom or sign had to be associated with an appropriate read-code. These issues were addressed with the help of the practice manager, who has much experience in the creation of such templates.

A draft of this new template was shown to colleagues at the practice. It was highlighted that a box-ticking exercise would not encourage the use of the template. It was suggested that the template include short explanation of the significance of particular findings. For example, the significance of whether or not the patient is a contact lens wearer was stated, as this may influence the choice of antibiotics. The final version of the eye template lists the six sight-threatening differentials, includes a checklist of the four red flag symptoms, contact lens status and common eye examinations (figure 6).

The final version of the electronic template was agreed and published. It can now be easily found within the practice's electronic patient record. As the Birchwood practice has a large number of trainees, from Foundation Year 2 level up, it is hoped that this would be a helpful reference guide for future trainees.

\section{Results}

The practice was re-audited six weeks after the practice meeting. Sixteen new cases had been read-coded "conjunctivitis". Pain was checked $63 \%$ of the time, compared to $26 \%$ prior to intervention. Visual acuity screening had increased to from $35 \%$ to $69 \%$. Photophobia was the most significantly increased metric, going from being documented only $6 \%$ of the time to now $63 \%$ of the time.

Documentation of whether the symptoms were unilateral or bilateral had also increased from $88 \%$ to $94 \%$ of consultations. A bar chart comparing cycle 1 and cycle 2 can be found in the supplementary material, labelled figure 3 . A run-chart was created to compare the month-to-month variation (figure 4). The percentage of consultations which checked for and documented all four symptoms increased from an average of $2.8 \%$ to $50 \%$.

This data was inputted into MiniTab Statistical Analysis Software, and a two-sample percentage defective test (based on Fisher's exact test) was carried out. This observed difference in checking all four symptoms was statistically significant with a p-value of less than 0.01

See supplementary file: ds4272.doc - "Cycle 2 Bar Chart, RunChart and Pictures"

\section{Lessons and limitations}

This project showed that significant improvements can be made with small and simple interventions. However, the interventions must be carefully designed to target the core of the problem. Communication with the GP partners, practice manager, and senior nurse practitioners was critical throughout the project.
A limitation to this study was the short re-audit period, due to the author changing rotations. Although there was a statistically significant difference before and after intervention, it is not clear if these changes will continue long-term. This is a common difficulty with quality improvement projects being led by junior doctors who change sites frequently.

It is hoped that the presence of the eye equipment kit, "P.A.L.S" acronym card and electronic acute eye consultation template will encourage future trainees rotating to Birchwood Medical Practice to keep up the current standard.

It is also possible that the change in behaviour was due to the knowledge that a re-audit was taking place. However, it is felt that whatever the motivation for the improvement, it still illustrates an understanding of what should be examined and documented in an acute eye consultation. It is hoped that this period of audit will be the start to good eye assessment habits.

Overall, it was felt that the changes made were both practical and cost-effective. The cost of one eye kit was only £3.50 in total, but could make the difference between a wrong diagnosis and a correct one.

\section{Conclusion}

This project has shed light on how general practitioners approach acute eye consultations. The initial audit indicated that the diagnosis of conjunctivitis was made somewhat instinctively. Screening for the red flag eye symptoms to exclude sightthreatening conditions was very rarely carried out. Visual acuity was rarely formally tested, as many of the consultation rooms did not have a Snellen chart. These are habits that could lead to adverse events, as was illustrated by the case of a missed corneal abrasion found within this audit.

The brief time allocated to ophthalmology in undergraduate medical training paired with the inconsistent access to eye equipment within each consultation room, were identified as the two major contributing factors to the development of these habits. The interventions were therefore designed to target the reasons behind these habits.

Following intervention, the percentage of consultations which screened for all four findings rose from $2.8 \%$ to $50 \%$. This was found to be statistically significant, with a p-value of less than 0.01 .

This step-change improvement at the practice showed that the doctors were willing to make changes to their behaviour. This result was a good example of how doctors want to and will do the best for their patients, given the right tools and information.

As these changes were cost-effective yet high-yield, they should be achievable in any general practice. It is hoped that more NHS general practices would adopt similar changes in the interest of improving the quality of acute eye consultations in primary care. 


\section{BMJ Quality Improvement Reports}

\section{References}

1. Statham MO, Sharma A, Pane AR. Misdiagnosis of acute eye diseases by primary health care providers: incidence and implications. Med J Aust 2008; 189(7): 402-4.

2. Sheldrick J, Vernon S, Wilson A. Study of diagnostic accord between general practitioners and an ophthalmologist. BMJ 1992; 304(6834): 1096.

3. Wilson A. The red eye: a general practice survey. J R Coll Gen Pract 1987; 37(295): 62-4.

4. Morrell D. Expressions of morbidity in general practice. BMJ 1971; 2(5759):454.

5. Sheldrick J, Wilson A, Vernon S, Sheldrick C. Management of ophthalmic disease in general practice. Brit J Gen Pract 1993; 43(376): 459-62.

6. NICE guidelines Clinical Knowledge Summaries (last revised September 2012). http://cks.nice.org.uk/red-eye

7. NICE guidelines Clinical Knowledge Summaries (last revised August 2012). Retrieved from http://cks.nice.org.uk/conjunctivitis-infective

8. Shuttleworth G, Marsh G. How effective is undergraduate and postgraduate teaching in ophthalmology? Eye 1997; 11(5): 744-50.

\section{Declaration of interests}

The author does not declare any competing interests.

\section{Acknowledgements}

I wish to thank Dr Rama Mark (GP partner and project mentor), Christine Thorpe (practice manager), Sara Norman (practice administrator), Nurse Heather Lilley, and the rest of the GP partners, registrars and foundation trainees for their support and guidance throughout this project. 